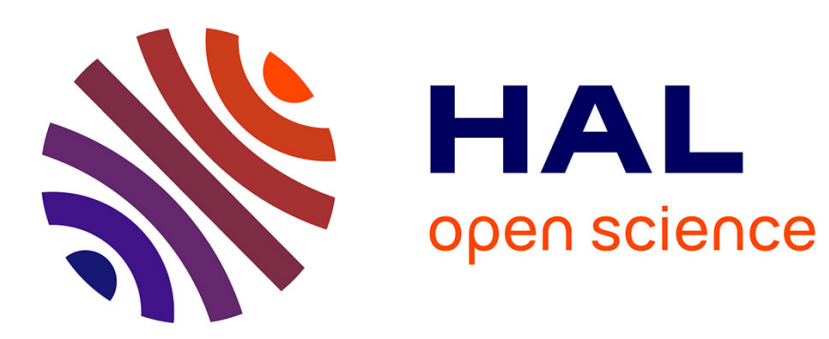

\title{
Single walled nanotubes/Amylose/SDBS complex
}

P. Bonnet, M. Gresil, H. Bizot, I. Riou, Bertoncini Patricia, A. Buleon,

Olivier Chauvet

\section{To cite this version:}

P. Bonnet, M. Gresil, H. Bizot, I. Riou, Bertoncini Patricia, et al.. Single walled nanotubes/Amylose/SDBS complex. 2009. hal-00363697

\section{HAL Id: hal-00363697 \\ https://hal.science/hal-00363697}

Preprint submitted on 24 Feb 2009

HAL is a multi-disciplinary open access archive for the deposit and dissemination of scientific research documents, whether they are published or not. The documents may come from teaching and research institutions in France or abroad, or from public or private research centers.
L'archive ouverte pluridisciplinaire HAL, est destinée au dépôt et à la diffusion de documents scientifiques de niveau recherche, publiés ou non, émanant des établissements d'enseignement et de recherche français ou étrangers, des laboratoires publics ou privés. 


\title{
Single walled nanotubes/Amylose/SDBS complex
}

\author{
P. Bonnet - M. Gresil - H. Bizot - I. Riou - P. Bertoncini - A. Buleon · \\ O. Chauvet
}

Received: date / Accepted: date

\begin{abstract}
In this work, we prepare and characterize single walled carbon/amylose complexes formed in aqueous medium in presence of a surfactant, where amylose is an helical polysaccharide. We show that at low amylose content, nanotubes based complexes are formed which show a strong infra-red fluorescence. Electron microscopy and atomic force microscopy suggest that the complex morphology implies also the surfactant. We propose a supramolecular architecture of the complex where the nanotubes are protected from the aqueous environment by the surfactant/amylose molecules. These complexes may be of interest for biosensing applications.
\end{abstract}

Keywords Nanotube $\cdot$ Polysaccharide Complex

\section{Introduction}

The formation of single walled carbon nanotubes (SWNTs) bio(macro)molecule complexes may open a broad range of applications in the biosensor field (Katz et al. 2004). It may also be helpful for the preparation of carbon nanotubes/biopolymer composites which can be used as structural biocompatible materials. A large number of complexes have already been prepared, for exemple complexes of SWNTs with proteins (Balavoine et al. 1999), peptides (Pantarotto et al. 2003), nucleic acids (Zheng et al. 2003), lipids (Richard et al. 2003) or enzyme (Besteman et al 2003). Some reports are also given about the formation of complexes between

P. Bonnet, M. Gresil, I. Riou, P. Bertoncini, O. Chauvet IMN, Universite de Nantes, CNRS, BP 32229, F-44322 Nantes, France

H. Bizot, A. Buleon

INRA BIA, BP 71627, F-44316 Nantes , France
SWNTs and polysaccharides such as arabic gum (Bandyopadhyaya et al. 2002), amylose or amylopectin (Star et al. 2002 , Stobinski et al. 2003), or $\beta$ - glucans (Numata et al. 2004). A potential interest of this last family comes from that it may allow further sugar chemistry. The nature of the interaction between biomolecules and SWNTs, whether it is a covalent functionalization, a $\pi$ stacking interaction or a wrapping of the macromolecule around the nanotube may be of importance for the foreseen applications. In case of SWNTs/polysaccharide system, inclusion complexes are usually inferred with a possible wrapping of the polymer chain around the SWNT (Xie et al. 2005). Indeed, such a "soft" functionnalization preserves the interesting intrinsic physico-chemical properties of the nanotubes. In this paper, we report the preparation of SWNT/amylose/surfactant complexes. The complexes are stable in aqueous solution and they show a strong near infra red fluorescence. We identify their supramolecular architecture which is stabilized by

s) $/ \pi$-stacking and hydrophobic interaction. These interactions represent thus an alternative to wrapping in order to disperse the nantoubes in aqueous environment.

\section{Complex preparation}

Amylose is a linear polymer of $\alpha(1-4)$ glucan which can complex various molecules such as lipids, alcohols, flavours (see Buleon et al. 2007 for a review). Depending on the guest molecule and the surrounding environment, amylose forms a sixfold (V6 conformation) or eightfold helix (V8 conformation) with an hydrophobic cavity whose the diameter is in the range of $0.55 \mathrm{~nm}$ to $0.9 \mathrm{~nm}$ (Buleon et al. 1998). In cold water and in absence of guest molecules, amylose chains usually self associate and form double helices without accessible in- 


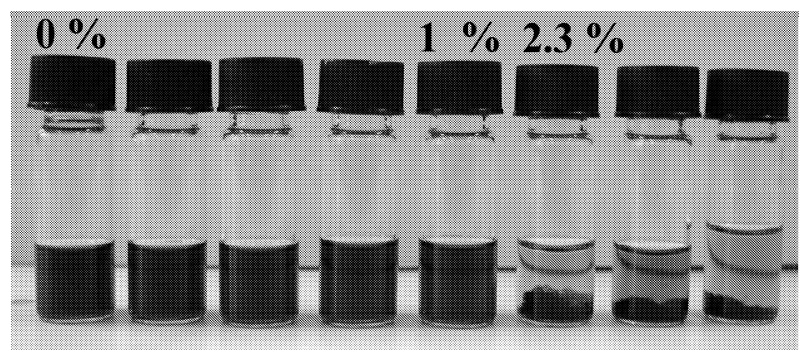

Fig. 1 SWNTs/ AmH dispersions in $\mathrm{D}_{2} \mathrm{O}$ with 0.7 wt $\%$ of SDBS. From left to right: $[\mathrm{AmH}]=0,0.1,0.2,0.5,1,2.3,3.3$ and $4.5 \mathrm{wt} \%$ respectively.

ternal gap. In order to avoid the double helix formation, we have prepared hydroxypropylated amylose $(\mathrm{AmH})$ where the 10-12\% hydroxypropyl side group per anhydroglucose unit inhibits the auto-association process (Wulff et al. 1998). The SWNTs/AmH complexes are obtained in aqueous solution in presence of sodium dodecyl benzenesulfonate (SDBS). Briefly, small diameter SWNTs (HipCo SWNTs from Carbon Nanotechnologies Inc.) are suspended in heavy water $(0.5 \mathrm{mg} / \mathrm{ml})$ with 0.7 wt \% SDBS followed by sonication and centrifugation. An amount of hydroxypropylated amylose of $[\mathrm{AmH}]=0$ to $[\mathrm{AmH}]=4.5 \mathrm{wt} \%$ is then added and mixed to the SWNTs suspension. More details are given elsewhere (Bonnet et al. 2007).

\section{Optical characterization}

As can be seen on Figure 1, stable suspensions are obtained as long as $[\mathrm{AmH}]$ does not exceed $2 \mathrm{wt} \%$ above which a partial precipitation occurs. More insights can be obtained by optical spectroscopies. Figure 2 shows the room temperature absorption spectra of suspensions with $[\mathrm{AmH}]=0,0.5$ and $3 \%$ respectively. Nicely resolved peaks are observed for 0 and $0.5 \%$ which are due to $E_{11}$ and $E_{22}$ optical transitions from well suspended and isolated SWNTs (O'Connell et al. 2002). It reveals that our preparation scheme succeeds in breaking the SWNTs bundles and in keeping the SWNTs isolated. Indeed, starting from isolated SWNTs is a prerequisite for their wrapping by amylose and conversely, an efficient wrapping is supposed to stabilize the dispersion. Emission spectra obtained with an excitation line at $752 \mathrm{~nm}\left(13299 \mathrm{~cm}^{-1}, 1.65 \mathrm{eV}\right)$ are shown in Figure $3(\mathrm{a})$ for $[\mathrm{AmH}]=0,0.5$ and $3 \%$ respectively. A zoom is given in Figure 3(b) for $[\mathrm{AmH}]=0.9 \%$ below 11000 $\mathrm{cm}^{-1}$. The sharp peaks are SWNTs Raman peaks while the broad features are due to NIR luminescence. The luminescence peaks (indicated by arrows) are observed below $2 \%$ of $\mathrm{AmH}$. These peaks, which are related to the $E_{11}$ transitions, are due to the radiative recombina-

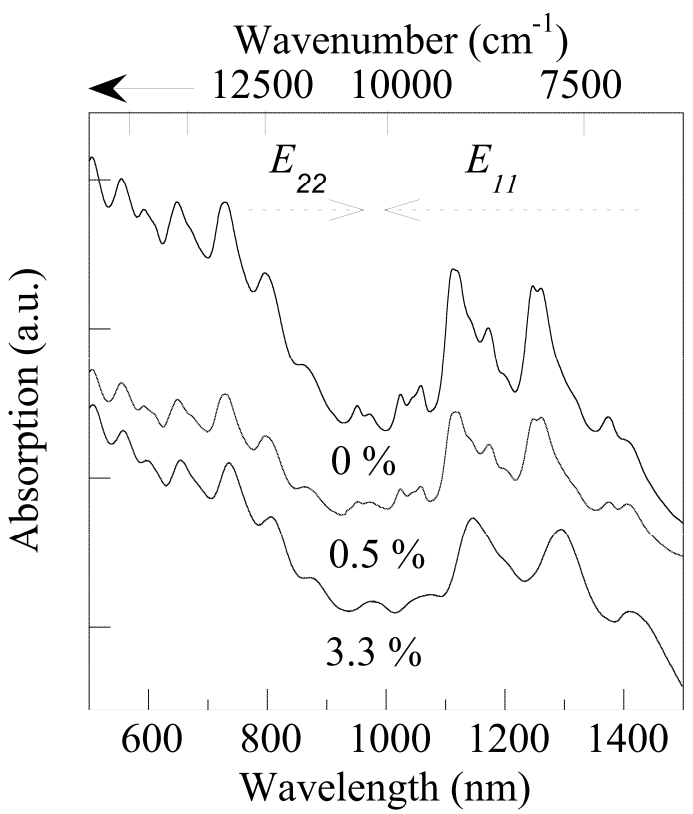

Fig. 2 Absorption spectra of SWNTs/AmH suspensions with $[\mathrm{AmH}]=0,0.5$ and $3 \%$ respectively. The wavelength range where $E_{11}$ and $E_{22}$ transitions are observed is marked with arrows.

tion of the excitons of semi-conducting individual tubes. Their position depends on the tube indexes. Following the assignment of the literature (Bachilo et al. 2002), the peaks of Figure 3(b) may be attributed to emission from $(9,4),(10,2),(7,5),(6,5),(8,3)$ and $(9,1)$ SWNTs respectively. All these tubes have diameters between 0.76 and $0.93 \mathrm{~nm}$ which is thus compatible with a possible insertion of the nanotube inside the internal gap of the amylose helix.

The behavior of the $[\mathrm{AmH}]=3.3 \%$ sample is quite different. A broadening and a red-shift of the $E_{11}$ absorption is observed on Figure 2 while the luminescence is quenched on Figure 3(a). It corresponds also to the destabilization of the suspension as shown on Figure 1. We will come back to these points later.

\section{Microscopic characterization}

While our spectroscopic investigations show that the SWNTs are isolated and well dispersed in solution when $[\mathrm{AmH}]<2 \%$, it is not clear whether it is due to a wrapping of amylose around the tubes or to another mechanism. Indeed, to answer this question is of importance since one may conjecture that the stabilization due to wrapping should be more robust than stabilization via another interaction mechanism. Transmission electron microscopy (TEM) at $200 \mathrm{keV}$ (Hitachi HF-2000 microscope) was performed on a $[\mathrm{AmH}]=0.2 \%$ sample. A typical TEM micrograph is shown on Figure 4. Most 


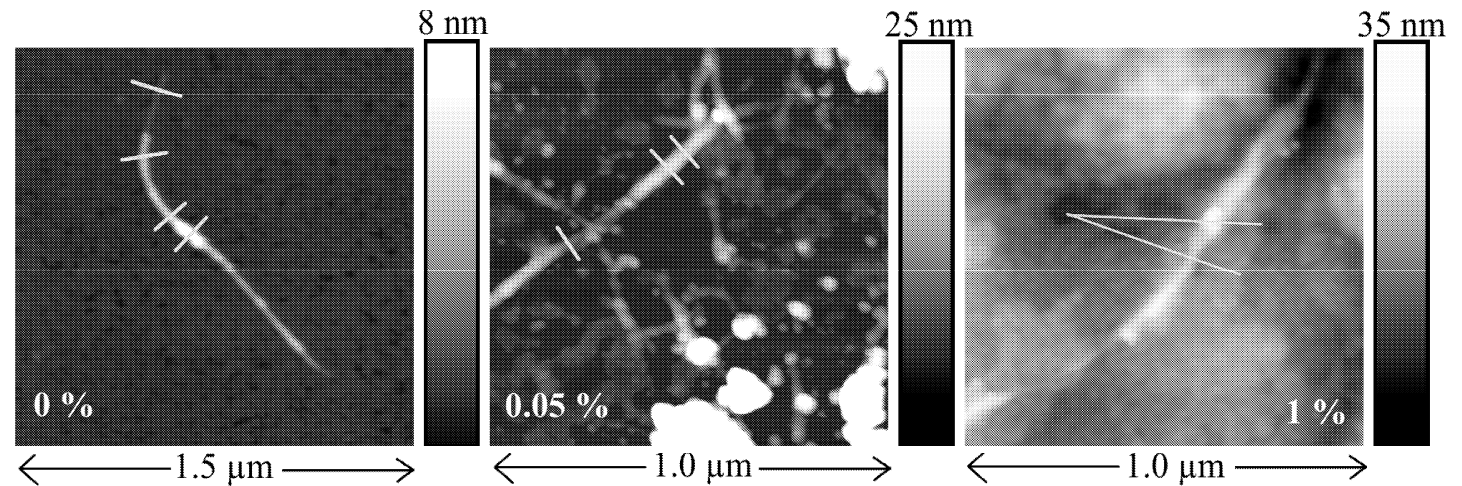

Fig. 5 AFM images of SWNT/AmH complex with (a) $[\mathrm{AmH}]=0 \mathrm{wt} \%$ - (b) $[\mathrm{AmH}]=0.05$ wt \% - (c) $[\mathrm{AmH}]=1$ wt \% - The lines indicate directions along which the height profiles were measured. Color on line

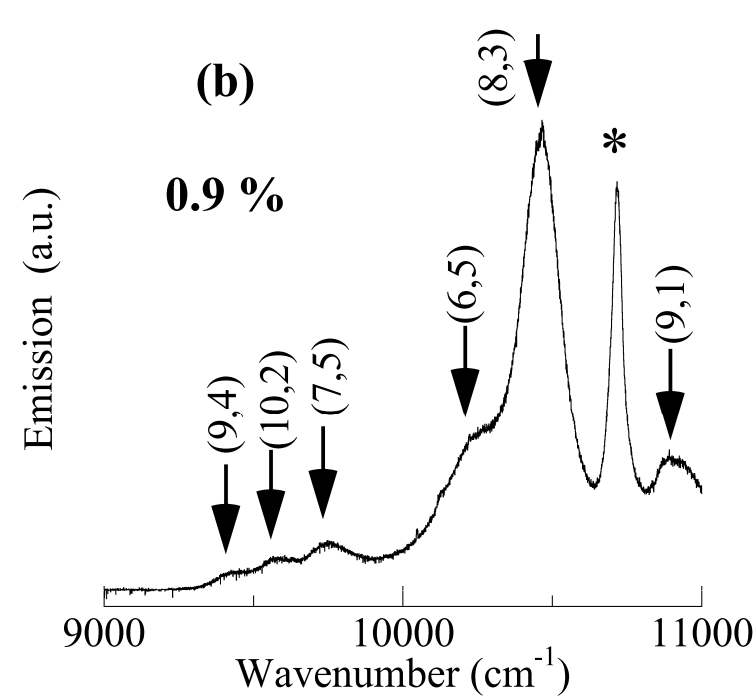

(a)

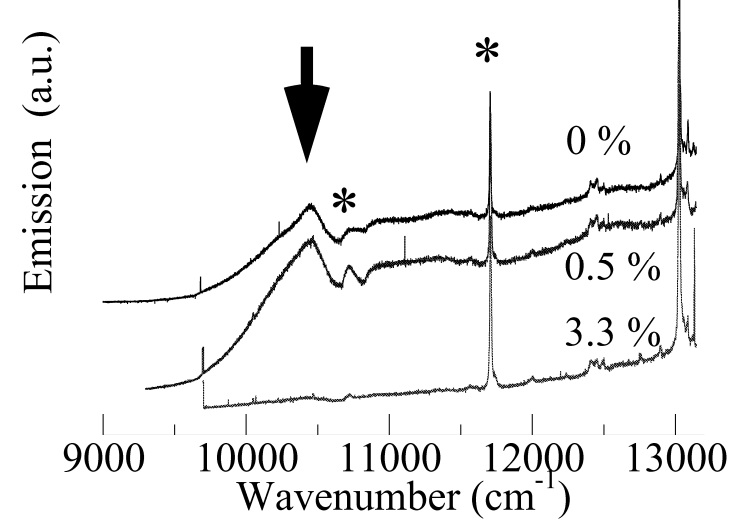

Fig. 3 (a) Emission spectra of SWNTs/AmH suspensions with $[\mathrm{AmH}]=0,0.5$ and $3.3 \mathrm{wt} \%$-(b) Details of an emission spectrum of a suspension with $[\mathrm{AmH}]=0.9 \mathrm{wt} \%$ - The arrows and the stars indicate fluorescence and Raman peaks of the SWNTs respectively - Excitation line : $752 \mathrm{~nm}(1.65 \mathrm{eV})$

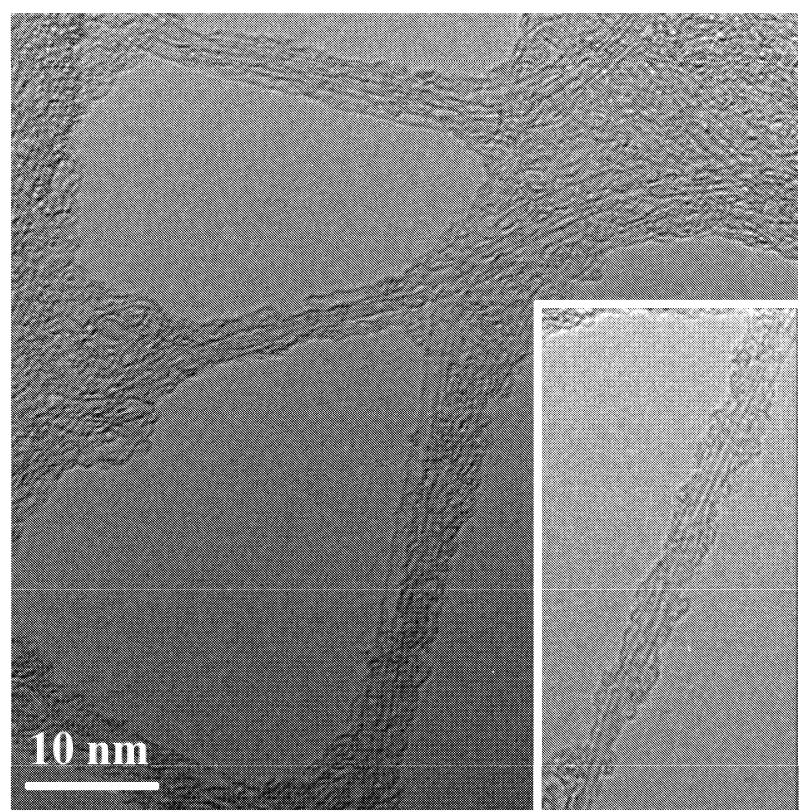

Fig. 4 TEM image of SWNT/AmH complex with $[\mathrm{AmH}]=0.2$ wt $\%$

of the SWNTs are isolated and they are surrounded by an organic layer. The chemical nature of this layer cannot be deduced from this observation and a wrapping or not is not evident.

Our samples were also investigated by Atomic Force Microscopy (Nanoscope IIIa from Digital Instruments) in tapping mode. The samples have been drop cast on a freshly clived mica surface and rinsed with distillated water in order to remove any excess of surfactant. Three typical sample images are shown on Figure 5 for $[\mathrm{AmH}]=0,0.05$ and $1 \%$ respectively. Despite the rinsing step, remaining surfactant micelles are observed on Figure $5(\mathrm{~b})([\mathrm{AmH}]=0.05 \%$; see the white globules $)$ while they are almost absent in the $[\mathrm{AmH}]=0 \%$ sample. Height profiles have been obtained along the lines drawn on the figure. For $[\mathrm{AmH}]=0 \%$, the nanotube 
height is measured at $1 \pm 0.5 \mathrm{~nm}$ (top line) while from top to bottom, the two next lines gives $4.0 \pm 0.5 \mathrm{~nm}$ and the last one $7.5 \pm 0.5 \mathrm{~nm}$. Indeed, the $1 \pm 0.5 \mathrm{~nm}$ height should correspond to a unique tube or a very small bundle while higher profiles are associated with SDBS covered part of this nanotube. In order to be more quantitative, we have deposited a SDBS solution of same concentration on a freshly cleaved graphite surface and on a mica surface. On graphite, two kinds of micelles are found with heights of $1.5 \pm 0.5 \mathrm{~nm}$ and $3.75 \pm 0.5 \mathrm{~nm}$ while only $3.5 \pm 0.5 \mathrm{~nm}$ high micelles are found on mica. It is noteworthy that this difference of behavior between SDBS deposited on graphite or on mica is not observed when SDS (sodium dodecylsulfonate) is used. In this last case, no difference in morphology and height of films are observable either on graphite or mica surface. It suggests that the SDBS molecules have a typical height of $3.5 \pm 0.5 \mathrm{~nm}$, and presumably hemi-micelles with the benzene ring oriented onto the hydrophobic graphite surface resulting in the reduced height observed for the first layer of SDBS molecules onto graphite. A direct comparison with results from Figure 5(a) suggests thus that a single SDBS hemi-micelle layer is covering the thinnest part of the tube while a typical double SDBS layer is covering the thicker part. A more precise structure of the complex is not resolved. When $[\mathrm{AmH}]=0.05 \%$, the situation is more complicated. From left to right, the average heights are respectively $5 \pm 1,14 \pm 1$ and $11 \pm$ $1 \mathrm{~nm}$. The organic layer is sensibly thicker in this sample. It is likely related to the presence of amylose around the tube. However, this thickness is much higher than what is expected for a nanotube simply wrapped by amylose. A geometrical sketch of such a wrapped nanotube by amylose is given in Figure 6 for which it can be seen that the thickness should be about $1.6 \mathrm{~nm}$ for the V6 helical conformation (6 glucose units per turn) while recently reported V8 complexes (8 glucose units per turn) offer cavities with inner diameter very close to the $\gamma$ cyclodextrin one of about $0.95 \mathrm{~nm}$ (Cardoso et al. 2007).

We are thus led to conclude that either there is much more than a single layer of amylose around the tube or that the SDBS has not been exchanged by amylose when we have inserted AmH into the solution. In turn, it is likely that AmH, SDBS and SWNTs form a three molecules complex in which SWNT is protected from water by an amylose/SDBS complex. We conjecture that the SWNTs/SDBS association is stabilized through a $\pi$-stacking interaction between the benzenoid head of the surfactant and the graphitic tube surface while the hydrophobic tail of the surfactant is inserted inside the amylose helix gap. In agreement with the

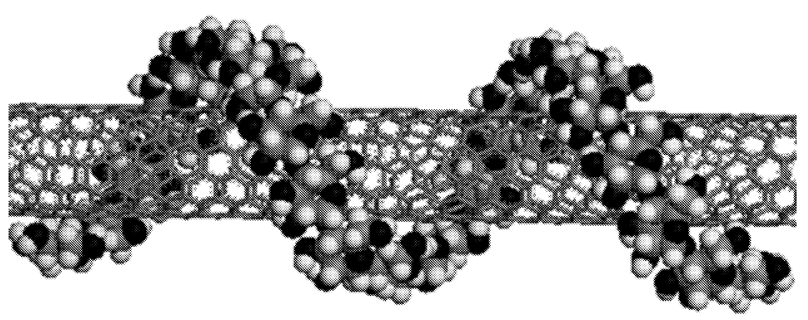

Fig. 6 Sketch of an amylose chain wrapping a $(6,6)$ SWNT. The amylose chain geometry is obtained from data extracted from ref.[6]- Atoms: Oxygen (Black), Carbon (Grey), Hydrogen (White)

observations, such a complex should be thicker than the SWNTs/SDBS complex of Figure 5(a). As shown in Figure 5(c), when $[\mathrm{AmH}]$ reaches 1 wt \%, hydroxypropylated amylose starts to form a film from which it is difficult to identify nanotubes. The typical height of the film by comparison with the mica surface (darker part of the image) is close to $10 \mathrm{~nm}$ here, and the line locations from bottom to top are at 14 and $20 \mathrm{~nm}$ high respectively. It suggests that the SWNT is covered by a layer of same nature than in the previous case. Above $1 \mathrm{wt} \%$ of amylose, the film deposited on mica is dense and smooth and it is no more possible to observe nanotubes.

\section{Discussion and Conclusion}

Let us briefly discuss the results. At low amylose content (below $<2 \%$ ), TEM and AFM images suggest that SWNTs/SDBS/AmH complexes are formed in which the SDBS has not been exchanged by AmH. We argue that the SDBS/nanotube interaction is mediated through a $\pi$-stacking between the benzenoid head of the surfactant and the nanotube surface while the hydrophobic tail of the surfactant is complexed with $\mathrm{AmH}$, which should protect the tail from the water environment. It is woth noticing that Sodium Dodecyl Sulfate (SDS) is able to form such kind of complex with Amylose (Nishimura et al. 1975). Despite its helical structure, the amylose chains do not wrap the tube. It explains why we do not observe any selectivity of the association: there is no significative differences in the absorption spectra of Figure 2 nor in the emission spectra before of after the addition of amylose. Above $2 \%$ of amylose, amylose chains are in large number, they associate into the solution and they form a film when deposited on the mica substrate. The SWNTs, still covered by the polymer are embedded in the film 
and they become closer. It leads to the broadening and the red shift of the $E_{11}$ absorption bands of Figure 2. In the same time, the fluorescence is partly quenched. It is likely due to a non radiative energy transfer from the tube to the amylose chains which are now in close contact or to a Forster antenna mechanism in direction of the metallic tubes. This organisation of the complex is quite different to what is suggested in the paper of Star et al. (Star et al. 2002). The presence of SDBS in the solution likely explains this difference.

In summary, we have prepared SWNTs/amylose complexes in aqueous solution useful to design active materials for biosensing. They show NIR fluorescent emission when the amylose content is less than $1 \%$ in aqueous solution, which is of interest for applications. The stabilization of the complex is not obtained by wrapping of the polysaccharide chain around the nanotube and the SDBS surfactant is also involved in a three molecules complex structure. Our investigations suggest that the hydrophobic tail of the surfactant is complexed with amylose while its benzenoid head interacts with the nanotube surface by $\pi$ - stacking. This complex morphology is not selective towards a specific tube geometry. Since wrapping is not necessary, linear or branched polysaccharide may be used to disperse the nanotubes as well. We are currently exploring the cellulose family.

\section{References}

1. Bachilo S M, Strano M S, Kittrell C, Hauge R H, Smalley R E, Weisman R B, (2002) Structure assigned optical spectra of single walled carbon nanotubes, Science 298:2361-2366

2. Balavoine F, Schultz P, Richard C, Mallouh V, Ebbesen T W, Mioskowski C, (1999) Helical Crystallization of Proteins on Carbon Nanotubes: A First Step towards the Development of New Biosensors, Angew. Chem. Int. Ed. 38:1912-1915

3. Bandyopadhyaya A, Nativ Roth E, Regev O, Yerushalmi Rozen R, (2002) Stabilization of Individual Carbon Nanotubes in Aqueous Solutions, Nanoletters 2:25-28

4. Besteman K, Lee J O, Wiertz F G, Heering H A, Dekker C, (2003) Enzyme-Coated Carbon Nanotubes as Single-Molecule Biosensors, Nanoletters 3:727-730

5. Bonnet P, Albertini D, Bizot H, Bernard A, Chauvet O, (2007) Amylose/SWNT composites: From solution to film Synthesis, characterization and properties, Composites Science and Technology $67: 817-821$

6. Buleon A, Colonna P, Planchot V, Ball S, (1998) Starch granules: structure and biosynthesis, Int. J. of Biol. Macromol. $23: 85-112$

7. Buleon A, Veronese G, Putaux J L, (2007) Self-Association and Crystallization of Amylose, Australian J. Chem. 60:706718

8. Cardoso M B, Putaux J L, Nishiyama Y, Helbert W, Hytch M, Silveira N P, Chanzy H, (2007) Single crystals of V-amylose complexed with alpha-naphthol, Biomacromol. 8:1319-1326

9. Katz E, Wilmer I, (2004) Biomolecule functionalized carbon nanotubes: applications in Nanobioelectronics, Chem. Phys. Chem. 5:1084
10. Nishimura N, Janado M, (1975) Gel Electrophoresis of Amylose in the Presence of Sodium Dodecyl Sulfate, J. Biochem 7:421-426

11. Numata M, Asai M, Kaneko K, Hasegawa H, Fulita N, Kitada Y, Sakurai K, Shinkai S, (2004) Curdlan and Schizophyllan ( $\beta$-1,3-Glucans) can Entrap Single-wall Carbon Nanotubes in Their Helical Superstructure, Chem. Letters 33:232

12. O'Connell M J, Bachilo S M, Huffman C B, Moore V C, Strano M S, Haroz E H, Rialon K L, Boul P J, Noon W H, Kittrell C, Ma J, Hauge R H, Weisman R B, Smalley R E, (2002) Band gap fluorescence from individual sinle walled carbon nanotubes, Science 297:593-596

13. Pantarotto D, Partidos C D, Graff R, Hoebeke J, Briand J P, Prato M, Bianco A , (2003) Synthesis, structural characterization, and immunological properties of carbon nanotubes functionalized with peptides, J. Am. Chem. Soc. 125:6160-6164

14. Richard C, Balavoine F, Schultz P, Ebbesen T W, Miokowski C, (2003) Supramolecular Self-Assembly of Lipid Derivatives on Carbon Nanotubes , Science 300:775-778

15. Star A, Steuerman D W, Heath J R, Stoddart J F, (2002) Starched carbon nanotubes, Angew. Chem. Int. Ed. 41:25082512

16. Stobinski L, Tomasik P, Lii C Y, Chan H H, Lin H M, Liu H L, Kao C T, Lu K S, (2003) Single-walled carbon nanotube amylopectin complexes, Carbohydr. Polym. 51:311-316

17. Wulff G, Steinert A, Hller O, (1998) Modification of amylose and investigation of its inclusion behavior, Carbohydr. Res. 307:19-31

18. Xie Y H, Soh A K, (2005) Investigation of non-covalent association of single-walled carbon nanotube with amylose by molecular dynamics simulation, Materials Letters 2:971-975

19. Zheng M, Jagota A, Semke A D, Diner B A, McLean R S, Lustig S R, Richardson R E, Tassi N G, (2003) DNA-assisted dispersion and separation of carbon nanotubes, Nature Mater. $2: 338-342$ 